\title{
Characterization of nanopores arrangement of anodic alumina layers synthesized on low-(AA1050) and high-purity aluminum by two-step anodizing in sulfuric acid with addition of ethylene glycol at low temperature
}

\author{
Marta Michalska-Domańska ${ }^{1}$ (D) Wojciech J. Stępniowski ${ }^{2}$ Leszek R. Jaroszewicz ${ }^{3}$
}

Published online: 4 November 2016

(c) The Author(s) 2016. This article is published with open access at Springerlink.com

\begin{abstract}
In this work the anodic aluminum oxide (AAO) was produced by two-step self-organized anodization of two classes purity aluminum in $0.3 \mathrm{M}$ sulfuric acid aqueous solution with addition of ethylene glycol as the solution modifier. The arrangement analysis method based on fast Fourier transforms was applied to characterize the porous arrays ordering. The quality, arrangement, circularity and regularity of nanoporous AAO formed on the low-purity (AA1050) and high-purity aluminum were investigated in details. It was found that the regularity of the nanopores ordering obtained on low-purity aluminum at applied experimental conditions was extraordinarily high while compared to the data published previously. The relation between the structural features of nanopores as well as aluminum concaves ordering obtained in two-step anodization process depending on the step of reaction, was analyzed. It was proven that purity of anodizing aluminum influence the interpore distance, wherein the purer aluminum the smaller interpore distance. The observed phenomenon was discussed in details in accordance with intrinsic mechanism. Furthermore, there are no simple relations between regularity ratio of obtained nanostructures and the step of anodizing.
\end{abstract}

Marta Michalska-Domańska

marta.michalska@wat.edu.pl; mrk.michalski@gmail.com

1 Institute of Optoelectronics, Military University of Technology, 2 Kaliskiego Str., 00-908 Warsaw, Poland

2 Department of Advanced Materials and Technologies, Faculty of Advanced Technology and Chemistry, Military University of Technology, 2 Kaliskiego Str., 00-908 Warsaw, Poland

3 Institute of Applied Physics, Military University of Technology, 2 Kaliskiego Str., 00-908 Warsaw, Poland
Keywords Anodic aluminum oxide (AAO) · Low-purity aluminum (AA1050) - Sulfuric acid · Fast Fourier transform - Regularity ratio - Interpore distance . Electrolyte modifier

\section{Introduction}

Nowadays the nanofabrication becomes one of the most important filed in the materials science and industry, because of unique, desirable and unusual properties of nanomaterials. One of the most commonly used approach of manufacture nanomaterials such as nanodots [1, 2], nanowires [3-7] and nanotubes [8,9] of metal, oxide or semiconductor $[10,11]$ is template assisted method with the use of anodic aluminium oxide (AAO) as a template. In this aspect the arrangement of nanopores of the nanoporous aluminium oxide template is very important parameter, because it determines the arrangement of the fabricated nanomaterials [12].

Since Masuda and Fukuda [13] in 1995 invented the self-organized two-step method for the production of wellarranged anodic aluminium oxide (AAO) template, researchers are still make efforts oriented on finding new two-step self-ordering anodization condition. Typically, the two-step anodization of aluminium is conducted in sulfuric $[14,15]$, oxalic [16, 17] and phosphoric [18, 19] acid aqueous solution at voltages in the range of $15-25,20-60$ and $120-195 \mathrm{~V}$, respectively. Depending on the anodization electrolyte, it is possible to obtain AAO membrane with cell diameter in the range of $25-75,75-125$, $375-525 \mathrm{~nm}$ in sulphuric, oxalic and phosphoric acid solution as electrolyte, respectively. According to research published recently, it is possible to extend this range up to $530-670 \mathrm{~nm}$ in cell diameter by using etidronic aqueous 
solution as novel self-ordering electrolyte and anodizing $\mathrm{Al}$ foils at voltage in the range $210-270 \mathrm{~V}$ at $0-60{ }^{\circ} \mathrm{C}$ [20]. What is more, additions of various modifiers to well know electrolytes [21] or to the new ones are recently reported in numerous papers [20, 22-24]. Usually the anodized aluminium is high purity $(99.99 \%)$, but there are many papers about anodized aluminum alloys [21, 25-29], because they are generally less expensive and more accessible then highpurity aluminum.

According to Masuda and Fukuda [13], during two-step anodization in the first step the disordered oxide is formed and concaves are generated at the metal-grown oxide interface. To obtain long-ordered arrangement of nanopores the second step of anodization is necessary. In this aim the nanoporous anodic aluminium oxide formed as the result of the first step of anodization is removed, the concaves are being revealed by selective chemical oxide removal and after that the second, providing highlyordered, concaves-based anodic aluminium oxide are formed. According to the two-step self-ordering aluminium anodization mechanism, the concaves formed during the first step of the reaction determine the quality of anodic alumina oxide formed after the second step of anodization. It suggests that the concaves and AAO obtained in second step of anodization should have comparable arrangement. In this paper we try to check this statement.

In this paper the arrangement analysis method based on fast Fourier transforms (FFT) was applied to investigate quantitatively arrangement of $\mathrm{AAO}$ formed in $0.3 \mathrm{M}$ $\mathrm{H}_{2} \mathrm{SO}_{4}$ with ethylene glycol (EG) as additive after the first step of anodization, concaves obtained after the oxide removal and arrangement of the final porous arrays. The relationship between arrangement, circularity and regularity ratio of AAO formed after the first step of anodizing, concaves obtained after the oxide removal and AAO formed after the second step of anodizing in sulphuric acid solution with EG as modifier were investigated in details. Additionally, the differences between nanopores arranging of anodic aluminum oxide formed on low-purity and highpurity aluminium were studied.

\section{Experimental}

Samples made of high-purity aluminum foil $(99.9995 \% \mathrm{Al}$, Puratronic, Alfa-Aesar) and commercially available lowpurity aluminum foil (99.5\%, AA1050) were prepared and anodized according the procedures described previously [21]. Briefly, the Al foils were cut into samples $(20 \times 20 \mathrm{~mm})$, next degreased in acetone and ethanol and subsequently electropolished (ethanol: $\mathrm{HClO}_{4}$ 1:4 vol. mixture, $10{ }^{\circ} \mathrm{C}, 0.5 \mathrm{~A} / \mathrm{cm}^{2}, 1 \mathrm{~min}$ ), rinsed with distilled water, ethanol and dried. A two-electrode electrochemical cell with $\mathrm{Pt}$ grid as cathode and Al samples as anode were used in the anodizing process. For each process a working surface of the sample and the distance between electrodes were kept constant and it were $1.14 \mathrm{~cm}^{2}$ and $1 \mathrm{~cm}$, respectively. The anodization was performed in a mixture of sulfuric acid solution (0.3 M), water and glycol (3:2 vol.) at various voltages $(15,20,25,30,35 \mathrm{~V})$ and at temperature of $-1{ }^{\circ} \mathrm{C}$ without stirring [1]. The addition of modifier fulfill the following roles in the processes: (1) allows to reduce anodization current, oxide growth rate and the heat of reaction, (2) thanks to higher viscosity of electrolyte allows to applied higher potentials in comparison to aqua sulfuric acid solution, (3) allows to carry out the reaction at low temperature without stirring of the electrolyte, (4) allows to applied higher maximum anodization which led to obtained larger pores than previously obtained in sulfuric acid solution without adding of
Table 1 The average pore diameter $\left(D_{\mathrm{p}}\right)$ and interpore distance (cell diameter, $D_{\mathrm{c}}$ ) of anodic aluminum oxide formed after the first step of anodization, concaves obtained after the oxide removal, and anodic aluminum oxide formed after the second step of anodization of high-purity and low-purity aluminum in $0.3 \mathrm{M}$ sulfuric acid with modulator at low temperature

\begin{tabular}{|c|c|c|c|c|c|}
\hline \multirow[t]{2}{*}{ Potential (V) } & \multicolumn{2}{|l|}{$D_{\mathrm{p}}$} & \multicolumn{3}{|l|}{$D_{\mathrm{c}}$} \\
\hline & 1st AAO & 2nd AAO & 1st AAO & Concaves & 2nd AAO \\
\hline \multicolumn{6}{|c|}{ High-purity aluminum } \\
\hline 15 & $17.5 \pm 4.0$ & $30.9 \pm 7.9[21]$ & $42.0 \pm 2.3$ & $40.9 \pm 0.4$ & $40.7 \pm 1.7[21]$ \\
\hline 20 & $23.9 \pm 4.9$ & $32.8 \pm 7.9[21]$ & $45.7 \pm 2.0$ & $51.3 \pm 0.4$ & $50.7 \pm 0.5[21]$ \\
\hline 25 & $22.8 \pm 4.6$ & $45.7 \pm 6.4[21]$ & $53.7 \pm 1.7$ & $59.9 \pm 0.7$ & $59.8 \pm 0.6[21]$ \\
\hline 30 & $23.7 \pm 6.0$ & $46.5 \pm 5.5[21]$ & $61.4 \pm 1.5$ & $68.5 \pm 0.6$ & $68.5 \pm 0.6[21]$ \\
\hline 35 & $22.5 \pm 6.0$ & $51.5 \pm 8.1[21]$ & $56.8 \pm 25.0$ & $77.9 \pm 1.1$ & $71.4 \pm 0.7[21]$ \\
\hline \multicolumn{6}{|c|}{ Low-purity aluminum } \\
\hline 15 & $20.2 \pm 3.2$ & $30.3 \pm 9.6[21]$ & $39.6 \pm 1.2$ & $41.3 \pm 1.3$ & $41.6 \pm 0.7[21]$ \\
\hline 20 & $24.1 \pm 7.1$ & $34.4 \pm 6.5[21]$ & $49.6 \pm 1.5$ & $51.2 \pm 0.8$ & $51.9 \pm 0.7[21]$ \\
\hline 25 & $21.3 \pm 3.7$ & $35.7 \pm 7.5[21]$ & $54.9 \pm 2.1$ & $60.1 \pm 0.6$ & $60.1 \pm 0.4[21]$ \\
\hline 30 & $23.6 \pm 4.2$ & $44.2 \pm 6.8[21]$ & $63.3 \pm 2.8$ & $70.3 \pm 0.9$ & $70.9 \pm 0.1[21]$ \\
\hline 35 & $26.6 \pm 9.6$ & $43.6 \pm 9.8[21]$ & $49.0 \pm 16.0$ & $80.7 \pm 1.3$ & $76.9 \pm 0.8[21]$ \\
\hline
\end{tabular}


Fig. 1 FE-SEM images (a, d, $\mathbf{g})$, their FFTs (b, e, h) with the intensity profiles $(\mathbf{c}, \mathbf{f}, \mathbf{i})$ for AAO after the first step of anodization $(\mathbf{a}-\mathbf{c})$, concaves on the aluminum surface, formed after oxide removal $(\mathbf{d}-\mathbf{f})$ and AAO after the second step of anodization $(\mathbf{g}-\mathbf{i})$ formed on low-purity aluminum in $0.3 \mathrm{M}$ sulfuric acid with ethylene glycol as solution modulator
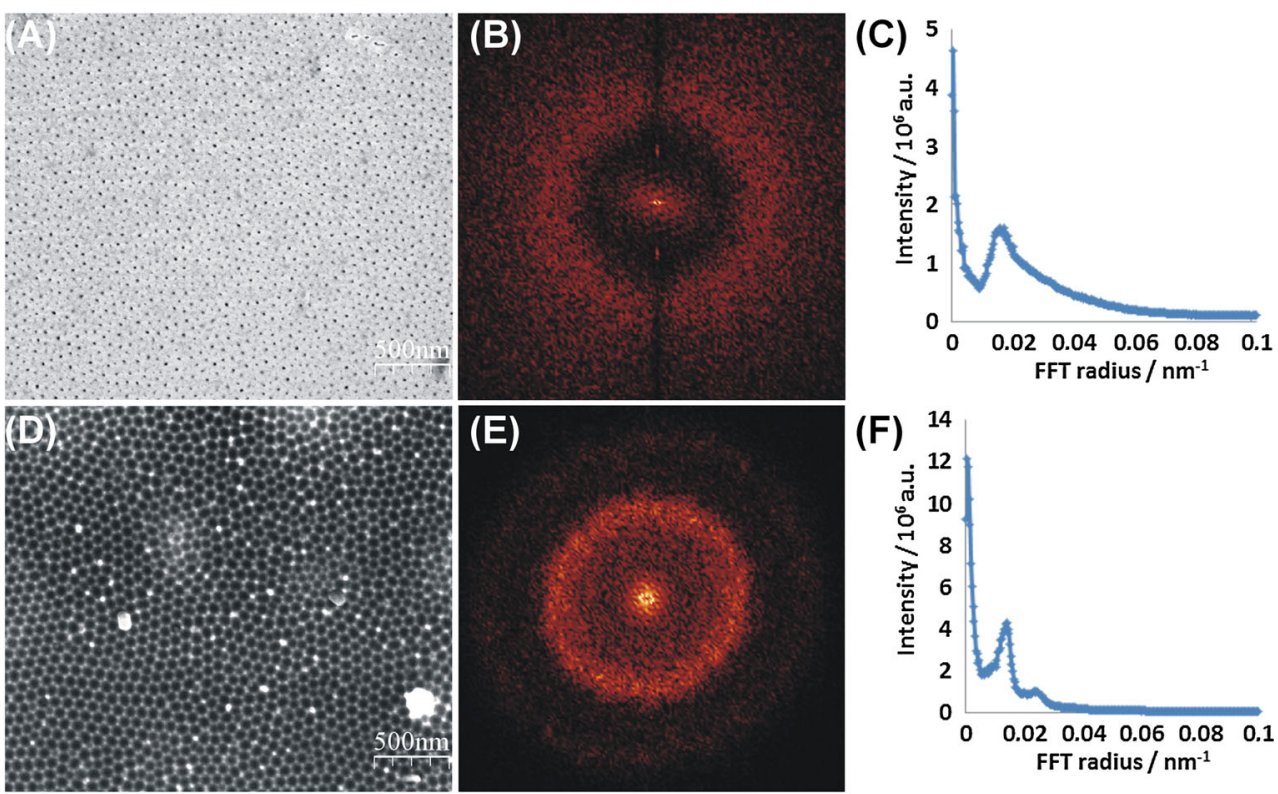

(F)
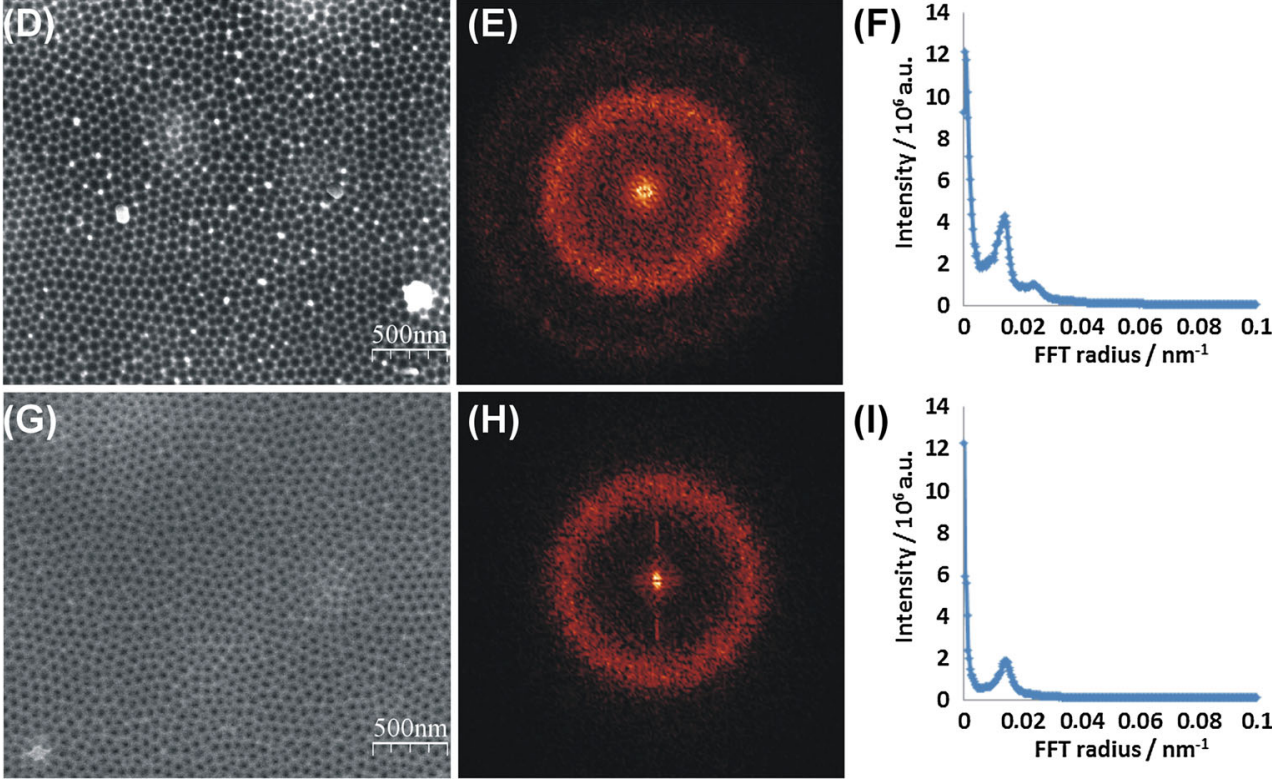

modifier [30]. The aluminum oxide formed after the 17-hlong first step of anodization was chemically etched in a mixture of $6 \mathrm{wt} \%$ phosphoric acid and $1.8 \mathrm{wt} \%$ chromic acid at $60{ }^{\circ} \mathrm{C}$ for $120 \mathrm{~min}$. To obtain highly-ordered nanoporous alumina the second anodization at the same set of operating conditions as applied in the first step for 30 min was performed. An adjustable DC power supply with voltage range of $0-60 \mathrm{~V}$ and current range of $0-5 \mathrm{~A}$, purchased from NDN model DF1760SL5A, was used to control the applied voltage.

Morphology images of fabricated nanoporous alumina were taken using field-emission scanning electron microscope FE-SEM (FEI, Quanta).

Fast Fourier transforms (FFT) were generated and taken into further calculations with WSxM software (WSxM 5.0 Develop 6.2) [31]. To define difference in quality of obtained AAO membranes the quantitative assessment of the hexagonal arrangement of nanopores in AAO, consisting the regularity ratio and circulatity, was performed. To estimate the regularity ratio, radial average, being a distribution function of inversed interpore distance, was generated from each FFT image. For every anodizing voltage three images at each step of experimental conditions were taken at the same magnification-100.000 $\times$. To estimate the regularity ratio, following equation was applied (1) [32]:

$R=\frac{I}{W} \cdot \frac{\sqrt{n}}{S^{\frac{3}{2}}}$

where $I$-is intensity of the radial average, $W$ is the width of the radial average at half of its height (FWHM), $n$ is the number of pores on the analyzed image and $S$ is analyzed surface area. Generally, higher value of regularity ratio meaning the better regularity of anodic aluminum oxide matrix. The better regularity of AAO template is important in many applications, for example in nanofabrication of nanostructures uses as metamaterials or photonic crystals.

Interpore distance was estimated as an inverse of the FFT's radial average [33]. The average interpore distance was estimated from three FE-SEM images for each sample. Pore diameter of the analyzed nanostructures was estimated from three FE-SEM images for each operating conditions, using NIS-Elements software.

The circularity was calculated for three images at the given set of experimental conditions. More information about geometrical parameters calculated methods was presented previously [32]. 
Fig. 2 FE-SEM images (a, d, $\mathbf{g})$, their FFTs $(\mathbf{b}, \mathbf{e}, \mathbf{h})$ with the intensity profiles $(\mathbf{c}, \mathbf{f}, \mathbf{i})$ for AAO after the first step of anodization $(\mathbf{a}-\mathbf{c})$, concaves on the aluminum surface, formed after oxide removal $(\mathbf{d}-\mathbf{f})$ and AAO after the second step of anodization $(\mathbf{g}-\mathbf{i})$ formed on high-purity aluminum in $0.3 \mathrm{M}$ sulfuric acid with ethylene glycol as solution modulator
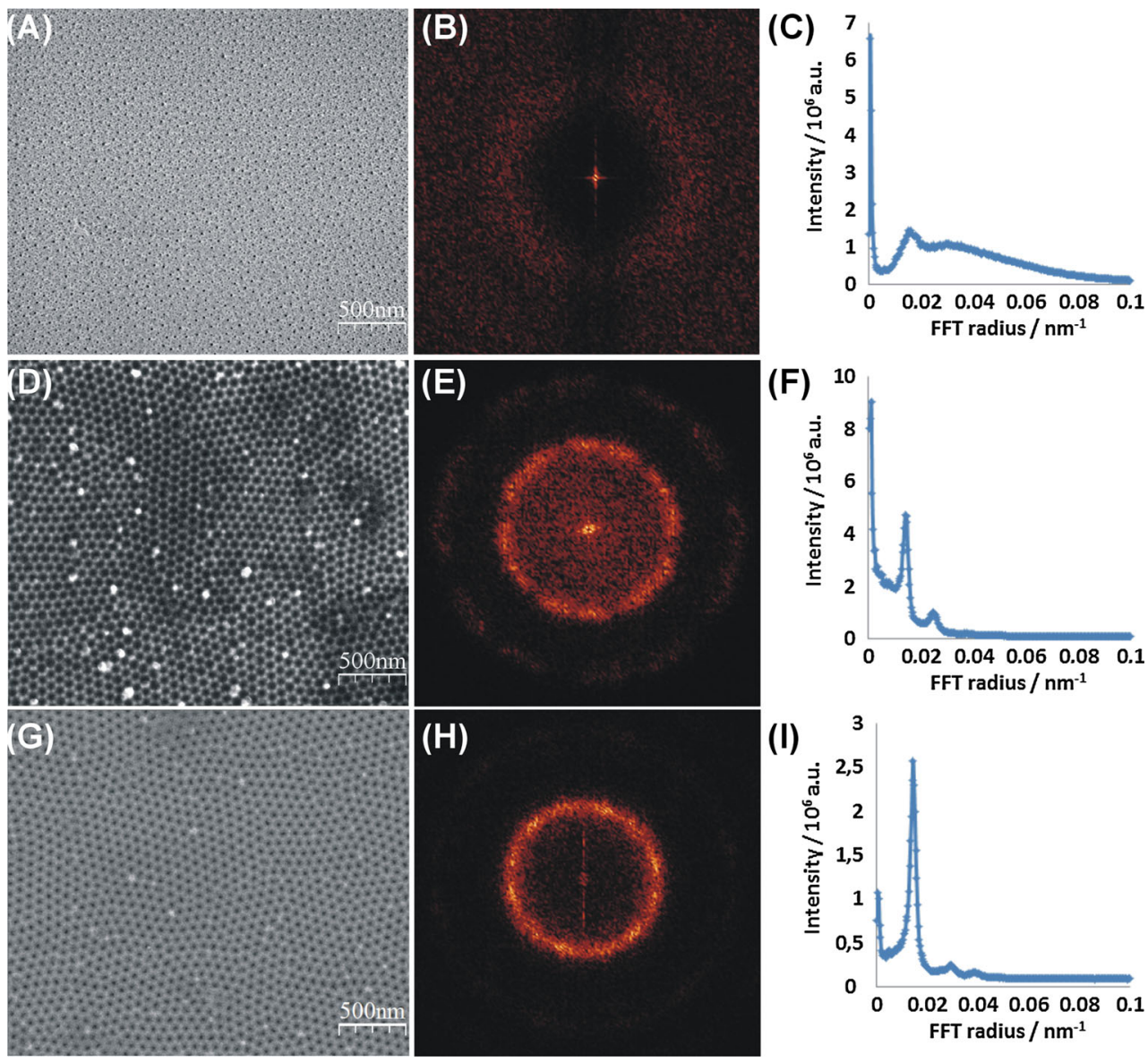

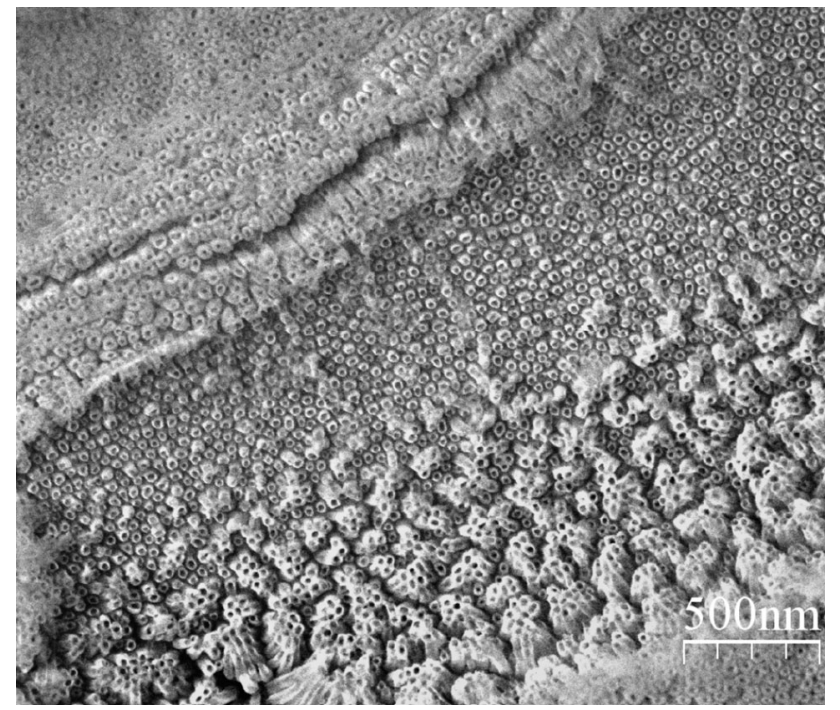

Fig. 3 The disordered morphology of AAO after second step of anodization formed on high-purity aluminum at $35 \mathrm{~V}$ (top side view)

\section{Results and discussion}

In Table 1 the geometrical features (pore diameter $D_{\mathrm{p}}$, interpore distance $D_{\mathrm{c}}$ ) of AAO formed after the first step of anodization, concaves after oxide removal and AAO formed after the second step of anodization are presented. The average pore diameter and interpore distance of AAO formed in both steps of anodization increase with applied voltage for high-purity as well as low-purity aluminum, which is in accordance with data presented before [21, 34-36]. The values of concaves interpore distance were similar to the values obtained for second AAO, what is expected according to the mechanism of aluminum twostep anodization. What is more, the $D_{\mathrm{c}}$ obtained for second step of anodization on low-purity aluminum was higher than on high-purity aluminum for all studied potentials. Many papers [37-40] proved that interpore distance are affected by type and concentration of electrolyte, applied voltage and reaction temperature. Although Kim et al. [41] analyzed the impact of purity of aluminum on the interpore distance showing no effect, but the difference with purity of aluminum was rather slight $(99.999 \%$ Al was comparable with $99.8 \% \mathrm{Al}$ ), thus any alloying element effect was negligible in this case. In this study the difference with purity of anodizing aluminum was relatively big: highpurity aluminum was $99.9995 \% \mathrm{Al}$ and low-purity aluminum was $99.5 \%$ Al. Moreover, all reaction parameters were fixed, so the differences in interpore distance were caused only by the purity of anodizing aluminum. It has 

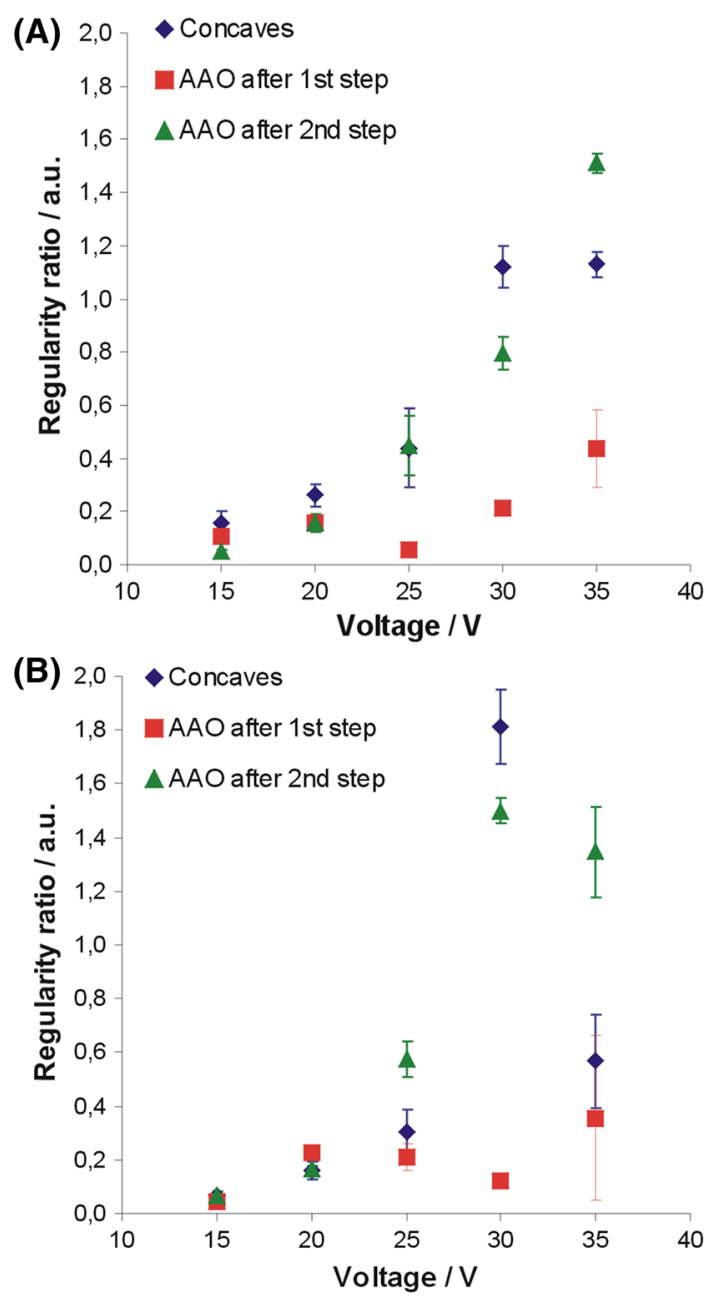

Fig. 4 Regularity ratio versus applied voltage for AAO formed after the first step of anodizing (red squares), concaves obtained after oxide removal (blue diamonds) and AAO formed after the second step of anodization (green triangles) obtained on low-purity (a) and highpurity (b) aluminum (Color figure online)

been known that the $D_{\mathrm{c}}$ increases with the applied potential [42-44]. Likewise anodization current density is made of ionic density $\left(i_{\mathrm{i}}\right)$ and electronic current density $\left(i_{\mathrm{e}}\right)$. It is commonly accepted that during the anodization process the ionic current density $\left(i_{\mathrm{i}}\right)$ can be further divided into oxidation current density $\left(i_{\mathrm{o}}\right)$ and incorporation current density $\left(i_{\mathrm{c}}\right)[42,43]$. The addition of EG to electrolyte change the viscosity and as a result decreased $i_{\mathrm{i}}$ and $i_{\mathrm{c}}$-this effect concerns to both examinated aluminum foils. On the other hand the impurities present in low-purity aluminum can increase $i_{\mathrm{e}}$ in compared to the high-purity aluminum during anodization, which can leads to receipt higher interpore distance in AAO made on low-purity aluminum foil.

The quantitative arrangement analysis, based on fast Fourier transform (FFT), was performed for AAO formed after first step of anodization, concaves revealed after the oxide removal and AAO formed after the second step of anodization on low-purity (Fig. 1) and high-purity (Fig. 2) aluminum. For each state the exemplary FE-SEM images (Figs. 1a, d, g, 2a, d, g), fast Fourier transforms (Figs. 1b, e, h, 2b, e, h) and their intensity profiles (Figs. 1c, f, i, 2c, f, i) were presented. For ideal hexagonal pore arrangement a FFT pattern consist of six points on the ring perimeter. When in the ideal arrangement the deviation or defects appears, the FFT ring becomes blurred or even disc-shaped. In this paper the analysis of FFTs images indicated that nanostructures obtained on high-purity aluminum in every step of anodization had better arrangement than respective nanostructures formed on low-purity aluminum. The most regular shapes of FFT ring and consequently best arrangement was observed after the second step of anodization, what was expected.

It was found that during anodization of high-purity aluminum at $35 \mathrm{~V}$ the AAO formed after the second step is deformed (Fig. 3). On the surface one can see nanostructures more similar to aluminum oxide nanotubes than nanopores. Additionally, on the AAO produced on highpurity aluminum at $35 \mathrm{~V}$ the crash and hillocks are present because of the incorporated $\mathrm{SO}_{4}{ }^{2-}$ ions, which has already been reported [21]. The presence of such structures resulted in decrease of regularity ratio for the AAO obtained on high-purity aluminum at $35 \mathrm{~V}$ after the second step of anodization (Fig. 4b).

The regularity ratio calculated for nanostructures obtained in each step of anodization in a function of applied potential was demonstrated in Fig. 4. Qualitatively, the slightly better arrangement of all obtained nanostructures was for high-purity aluminum than for low-purity aluminum (compare Fig. 4a, b). On the other hand those differences in regularity ratio values between AAO template made on high-purity and low-purity aluminum with comparing to the lower cost of low-purity aluminum were not so significant and this is fully acceptable. For anodization carried out on low-purity aluminum, the highest regularity ratio was observed for second AAO formed at $35 \mathrm{~V}$. Importantly, the nanostructures formed on low-purity aluminum are characterized with a very high regularity, which is quite extraordinary compared to the data published before [30]. In contrast, for anodization carried out on high-purity aluminum, the highest regularity ratio was observed for concaves produced at $30 \mathrm{~V}$. As it was mentioned above, at higher voltages the ordering of nanopores becomes worse because of the crash and hillocks formation on the AAO surface [21]. For low anodizing voltage the regularity ratio AAO formed after first step of anodization, concaves and AAO formed after the second step of anodization, are comparable. The arrangement of AAO obtained on high-purity aluminum at $20 \mathrm{~V}$ after the first step of anodization is better than arrangement of the concaves or AAO formed after the second step of 
Table 2 Circularity of anodic aluminum oxide formed after the first step of anodizing, concaves after the oxide removal and anodic aluminum oxide formed after the second step of anodizing in $0.3 \mathrm{M}$ sulfuric acid with glycol as modulator at temperature of $-1{ }^{\circ} \mathrm{C}$ for various anodizing voltage on high-purity and low-purity aluminum

\begin{tabular}{|c|c|c|c|c|c|c|}
\hline \multirow[t]{2}{*}{ Potential (V) } & \multicolumn{3}{|c|}{ High-purity aluminum } & \multicolumn{3}{|c|}{ Low-purity aluminum } \\
\hline & 1st AAO & Concaves & 2nd AAO & 1st AAO & Concaves & 2nd AAO \\
\hline 15 & $0.22 \pm 0.16$ & $0.26 \pm 0.13$ & $0.78 \pm 0.21$ & $0.61 \pm 0.24$ & $0.46 \pm 0.24$ & $0.59 \pm 0.27$ \\
\hline 20 & $0.44 \pm 0.20$ & $0.23 \pm 0.11$ & $0.18 \pm 0.10$ & $0.48 \pm 0.22$ & $0.29 \pm 0.14$ & $0.38 \pm 0.18$ \\
\hline 25 & $0.54 \pm 0.26$ & $0.16 \pm 0.11$ & $0.14 \pm 0.09$ & $0.59 \pm 0.25$ & $0.34 \pm 0.12$ & $0.19 \pm 0.09$ \\
\hline 30 & $0.68 \pm 0.24$ & $0.20 \pm 0.10$ & $0.16 \pm 0.06$ & $0.56 \pm 0.16$ & $0.33 \pm 0.11$ & $0.14 \pm 0.06$ \\
\hline 35 & $0.56 \pm 0.25$ & $0.21 \pm 0.09$ & $0.23 \pm 0.13$ & $0.08 \pm 0.06$ & $0.34 \pm 0.11$ & $0.33 \pm 0.13$ \\
\hline
\end{tabular}

anodization. Above $25 \mathrm{~V}$ the ordering of concaves and AAO formed after the second step of anodization is much better than for AAO obtained after the first step of anodization, what is in accordance with the mechanism of two-step aluminum anodization. However the arrangement of concaves and AAO formed after the second step of anodization change in unpredictable way. Notwithstanding, there is no simple relation between regularity ratio of obtained nanostructures and the step of anodizing. It is well know that the greater voltage leads to better arrangement of AAO nanopores. According $10 \%$ porosity rule provides by Nielsch et al. [44] the anodization of aluminum under optimum self-ordering condition leading to a quasiperfect hexagonal arrangement of pores. For mild anodization process conducted at $10{ }^{\circ} \mathrm{C}$ in $0.3 \mathrm{M}$ sulfuric acid used as electrolyte the optimum self-ordering voltage is $25 \mathrm{~V}$ [45-47]. In this research the mixture of sulfuric acid and EG as electrolyte was used, which allows to extend the range of applied anodizing voltage (from $15 \mathrm{~V}$ up to $35 \mathrm{~V}$ ) without burning phenomena occurs. According to intrinsic mechanism the modification of electrolyte by addition of ethylene should leads to decrease of dissociations constant, which leads to higher maximum anodization voltage $[42,43]$ and the better arrangement as consequence. However, above a critical applied anodizing potential, on the aluminum oxide the hillocks and cracks appears resulting in lower quality of AAO matrix [21].

One of the parameter to characterize the ideality of the obtained nanopores is circularity $(C)$, which can be estimated according to the following Eq. (2):

$C=\frac{4 \pi \cdot S}{L^{2}}$

where $S$ is a surface area occupied by single pore and $L$ is a perimeter of pore. When the pore is an ideal circle the $C$ equals 1.0 , but if the pore deformation occurs the $C$ value decrease and when pore is similar to elongated polygon the $C$ value is close to 0 . The average pore circularities calculated for all investigated sample was given in Table 2.
The highest circularities were noticed for AAO formed after the second step of anodization on high-purity aluminum at $15 \mathrm{~V}$ and for AAO after the first step of anodization produced on low-purity aluminum at $15 \mathrm{~V}$, which was confirmed by SEM observation but was incoherent with calculated regularity ratio (Fig. 4). The majority of calculated circularity was rather low values, independently from the step of anodizing reaction, wherein the nanostructures were formed. It should be remembered that the two-step anodization was performed at low temperature $\left(-1{ }^{\circ} \mathrm{C}\right)$, which resulted lower reaction rate and consequently, lower porosity, circularity and finally less regular shape of nanopores.

\section{Conclusion}

In summary, the slight better arrangement of nanopores and concaves were for high-purity aluminum than for low-purity aluminum for every step of anodization conducted up to $30 \mathrm{~V}$, but the differences were not significant. At certain experimental conditions the regularity of nanopores obtained on low-purity aluminum was extraordinary high. The arrangement of nanopores was generally better for AAO formed after the second step of anodization than for the oxide formed after the first step of anodization, especially for nanostructures obtained at anodization potential above $20 \mathrm{~V}$ for low-purity as well as high-purity aluminum. The arrangement of concaves and AAO formed after the second step of anodization obtained on both starting materials (regardless of aluminum foil purity) increase with applied potential up to $30 \mathrm{~V}$. The arrangement of the oxide obtained after the second step of anodization formed on high-purity aluminum at $35 \mathrm{~V}$ was surprisingly low, due to the formation mainly of nanotubes, instead of the nanopores. It was found that interpore distance was affected by purity of anodizing aluminum: $D_{\mathrm{c}}$ was greater for nanostructures obtained on low-purity aluminum than on high-purity aluminum for all tested 
experimental conditions. There is no clear relationship between arrangement of the anodic oxide formed after the first step of anodization, concaves and the oxide formed after the second step of anodization as it could be expected.

Acknowledgements This work was financially supported by the Polish Ministry of Science and Higher Education, Key Project POIG.01.03.01-14-016/08 "New Photonic Materials and their Advanced Applications", which is gratefully acknowledged. M. Michalska-Domańska acknowledges the financial support from the National Science Centre, Poland (Decision Number: DEC-2015/ 17/D/ST8/02432). W.J. Stępniowski acknowledges financial support from Foundation for the Polish Science (START 104.2014).

Open Access This article is distributed under the terms of the Creative Commons Attribution 4.0 International License (http://crea tivecommons.org/licenses/by/4.0/), which permits unrestricted use, distribution, and reproduction in any medium, provided you give appropriate credit to the original author(s) and the source, provide a link to the Creative Commons license, and indicate if changes were made.

\section{References}

1. M. Wu, U. Wen, Y. Lei, S. Ostendorp, K. Chen, G. Wild, Ultrathin alumina membranes for surface nanopatterning in fabricating quantum-sized nanodots. Small 6, 695-699 (2005)

2. Z. Fang, Y. Wang, X. Peng, X. Liu, C. Zhen, Structural and optical properties of $\mathrm{ZnO}$ films grown on the AAO templates. Mater. Lett. 57, 4187-4190 (2003)

3. Y.S. Cho, Y.D. Huh, Synthesis of ultralong copper nanowires by reduction of copper-amine complexes. Mater. Lett. 63, 227-229 (2009)

4. G. Song, X. Li, Y. Wang, Z. Peng, Y. Yu, P. Li, Patterned procedure for template-synthesis and microstructural characterization of copper nanowires. Mater. Charact. 61, 371-375 (2010)

5. X. Ren, Z. Lun, Mesoporous silica nanowires synthesized by electrodeposition in AAO. Mater. Lett. 68, 228-229 (2012)

6. R. Fathi, S. Sanjabi, N. Bayat, Synthesis and characterization of NiMn alloy nanowires via electrodeposition in AAO template. Mater. Lett. 66, 346-348 (2012)

7. A. Ghaddar, J. Gieraltowski, F. Gloaguen, Effects of anodization and electrodeposition conditions on the growth of copper and cobalt nanostructures in aluminum oxide films. J. Appl. Electrochem. 39, 719-725 (2009)

8. K. Yu, T. Luo, Y. Zhang, C. Yang, L. Shang, C. Li, Q. Li, Carbon nanotube synthesis over glow discharge-treated Ni/AAO membrane. Mater. Lett. 63, 566-568 (2009)

9. X. Ren, C.H. Jiang, D.D. Li, L. He, Fabrication of $\mathrm{ZnO}$ nanotubes with ultrathin wall by electrodeposition method. Mater. Lett. 62, 3114-3116 (2008)

10. Z. Bao, Y. Wu, $\mathrm{TiO}_{2}$ thin layer coated Ag nanoarrays complex for surface-enhanced Raman Scattering substrate. Key Eng. Mater. 562-565, 1037-1042 (2013)

11. C. Toccafondi, S. Thorat, R. La Rocca, A. Scarpellini, M. Salerno, S. Dante, G. Das, Multifunctional substrates of thin porous alumina for cell biosensors. J. Mater. Sci. Mater. Med. 25, 2411-2420 (2014)

12. W.J. Stępniowski, Z. Bojar, Nanoporous anodic aluminum oxide: fabrication, characterization, and applications, in Handbook of Nanoelectrochemistry: Electrochemical Synthesis Methods, Properties, and Characterization Techniques Part II, ed. By
M. Aliofkhazraei, A.S.H. Makhlouf, (Springer International Publishing Switzerland, 2016), pp. 593-646

13. H. Masuda, K. Fukuda, Ordered metal nanohole arrays made by a two-step replication of honeycomb structures of anodic alumina. Science 268, 1466-1468 (1995)

14. K. Schwirn, W. Lee, R. Hillebrand, M. Steinhart, K. Nielsch, U. Gösele, Self-ordered anodic aluminum oxide formed by $\mathrm{H}_{2} \mathrm{SO}_{4}$ hard anodization. ACS Nano 2(2), 302-310 (2008)

15. Y. Chen, A. Santos, D. Ho, Y. Wang, T. Kumeria, J. Li, C. Wang, D. Losic, On the generation of interferometric colors in high purity and technical grade aluminum: an alternative green process for metal finishing industry. Electrochim. Acta 174, 672-681 (2015)

16. A. Keshavarz, Z. Parang, A. Nasseri, The effect of sulfuric acid, oxalic acid, and their combination on the size and regularity of the porous alumina by anodization. J. Nanostruct. Chem. 3, 34 (2013)

17. W.J. Stępniowski, A. Nowak-Stępniowska, Z. Bojar, Quantitative arrangement analysis of anodic alumina formed by short anodizations in oxalic acid. Mater. Charact. 78, 79-86 (2013)

18. F. Le Coz, L. Arurault, L. Datas, Chemical analysis of a single basic cell of porous anodic aluminium oxide templates. Mater. Charact. 61, 283-288 (2010)

19. L. Zaraska, G.D. Sulka, M. Jaskuła, The effect of n-alcohols on porous anodic alumina formed by self-organized two-step anodizing of aluminum in phosphoric acid. Surf. Coat. Technol. 204, 1729-1737 (2010)

20. T. Kikuchi, O. Nishinaga, S. Natsui, R.O. Suzuki, Fabrication of self-ordered porous alumina via etidronic acid anodizing and structural color generation from submicrometer-scale dimple array. Electrochim. Acta 156, 235-243 (2015)

21. M. Michalska-Domańska, M. Norek, W.J. Stępniowski, B. Budner, Fabrication of high quality anodic aluminum oxide (AAO) on low purity aluminum - a comparative study with the AAO produced on high purity aluminium. Electrochim. Acta 105, 424-432 (2013)

22. T. Kikuchi, D. Nakajima, J. Kawashima, S. Natsui, R.O. Suzuki, Fabrication of anodic porous alumina via anodizing in cyclicoxocarbon acids. Appl. Surf. Sci. 313, 276-285 (2014)

23. T. Kikuchi, T. Yamamoto, S. Natsui, R.O. Suzuki, Fabrication of anodic porous alumina by squaric acid anodizing. Electrochim. Acta 123, 14-22 (2014)

24. T. Kikuchi, O. Nishinaga, S. Natsui, R.O. Suzuki, Self-ordering behavior of anodic porous alumina via selenicacid anodizing. Electrochim. Acta 137, 728-735 (2014)

25. W.J. Stępniowski, M. Michalska-Domańska, M. Norek, E. Twardosz, W. Florkiewicz, W. Polkowski, D. Zasada, Z. Bojar, Anodization of cold deformed technical purity aluminum (AA1050) in oxalic acid. Surf. Coat. Technol. 258, 268-274 (2014)

26. T. Shih, P. Wei, Y. Huang, Optical properties of anodic aluminum oxide films on Al1050 alloys. Surf. Coat. Technol. 202, 3298-3305 (2008)

27. C.S. Chi, J.H. Lee, I. Kim, H.J. Oh, Effects of microstructure of aluminum substrate on ordered nanopore arrays in anodic alumina. J. Mater. Sci. Technol. 31, 751-758 (2015)

28. D. Lo, R.A. Budiman, Fabrication and characterization of porous anodic aluminafilms from impure aluminum foils. J. Electrochem. Soc. 154(1), C60-C66 (2007)

29. L. Zaraska, E. Wierzbicka, E. Kurowska-Tabor, G.D. Sulka, Synthesis of nanoporous anodic alumina by anodic oxidation of low purity aluminum substrates, in Nanoporous Alumina, Springer Ser Mater Sci 219, ed. By D. Losic, A. Santos, (Springer International Publishing AG Switzerland is part of Springer Science+Business Media 2015), pp. 61-106 
30. L. Zaraska, G.D. Sulka, J. Szeremeta, M. Jaskuła, Porous anodic alumina formed by anodization of aluminum alloy (AA1050) and high purity aluminum. Electrochim. Acta 55, 4377-4386 (2010)

31. I. Horcas, R. Fernandez, J.M. Gomez-Rodriguez, J. Colchero, J. Gomez- Herrero, A.M. Baro, WSXM: a software for scanning probe microscopy and a tool for nanotechnology. Rev. Sci. Instrum. 78, 013705 (2007)

32. A. Nowak-Stępniowska, A review of quantitative arrangement analysis methods applied to nanostructured anodic oxides characterization. Curr. Nanosci. 11(5), 581-592 (2015)

33. M. Almasi Kashi, A. Ramazani, The effect of temperature and concentration on the self-organized pore formation in anodic alumina. J. Phys. D Appl. Phys. 38, 2396-2399 (2005)

34. G.D. Sulka, W.J. Stępniowski, Structural features of self-organized nanopore arrays formed by anodization of aluminum in oxalic acid at relatively high temperatures. Electrochim. Acta 54, 3683-3691 (2009)

35. L. Zaraska, W.J. Stępniowski, G.D. Sulka, E. Ciepiela, M. Jaskuła, Analysis of nanopore arrangement and structural features of anodic alumina layers formed by two-step anodizing in oxalic acid using the dedicated executable software. Appl. Phys. A 114, 571-577 (2014)

36. T. Kikuchi, D. Nakajima, O. Nishinaga, S. Natsusi, R.O. Suzuki, Porous aluminium oxide formed by anodizing in various electrolyte species. Curr. Nanosci. 11(5), 560-571 (2015)

37. L. Zaraska, W.J. Stępniowski, E. Ciepiela, G.D. Sulka, The effect of anodizing temperature on structural features and hexagonal arrangement of nanopores in alumina synthesized by two-step anodizing in oxalic acid. Thin Solid Films 534, 155-161 (2013)

38. W.J. Stępniowski, A. Nowak-Stępniowska, A. Presz, T. Czujko, R.A. Varin, The effects of time and temperature on the arrangement of anodic aluminium oxide nanopores. Mater. Charact. 91, 1-9 (2014)
39. H.B. Jiang, C.Z. Li, Y. Zhao, Effects of applied potential on the growth of porous aluminum oxide membrane. Chin. J. Process Eng. 2, 409-413 (2007)

40. X.-J. Liu, L.-F. Li, Y. Ren, Progress in research on the interpore distance of anodic aluminum oxide. Gongneng Cailiao/J. Funct. Mater. 44(22), 3225-3229 (2013)

41. B. Kim, J.S. Lee, Effect of aluminum purity on the pore formation of porous anodic alumina. Bull. Korean Chem. Soc. 35(2), 349-352 (2014)

42. L. Yi, L. Zhiyuan, H. Xing, L. Yisen, C. Yi, Investigation of intrinsic mechanisms of aluminum anodization processes by analyzing the current density. RSC Adv. 2, 5164-5171 (2012)

43. Z. Ling, Y. Li, Mechanisms of nanoporous alumina formation and self-organized growth, in Nanoporous Alumina, Springer Ser Mater Sci 219, ed. By D. Losic, A. Santos, (Springer International Publishing AG Switzerland is part of Springer Science+Business Media 2015), pp. 1-29

44. K. Nielsch, J. Choi, K. Schwirn, R.B. Wehrspohn, U. Gösele, Self-ordering regimes of porous alumina: the $10 \%$ porosity rule. Nano Lett. 2, 677-680 (2002)

45. H. Masuda, F. Hesegwa, S. Ono, Self-ordering of cell arrangement of anodic porous alumina formed in sulfuric acid solution. J. Electrochem. Soc. 144, L127-L130 (1997)

46. A.P. Li, F. Müller, A. Birner, K. Nielsch, U.J. Gösele, Hexagonal pore arrays with a $50-420 \mathrm{~nm}$ interpore distance formed by selforganization in anodic alumina. Appl. Phys. 84, 6023-6026 (1998)

47. A.P. Li, F. Müller, A. Birner, K. Nielsch, U. Gösele, Fabrication and microstructuring of hexagonally ordered two dimensional nanopore arrays in anodic alumina. J. Vac. Sci. Technol. A 17, 1428-1431 (1999) 